\title{
Effect of phospholipids and their molecular species on cholesterol solubility and nucleation in human and model biles
}

\author{
Z Halpern, M Moshkowitz, H Laufer, Y Peled, T Gilat
}

\begin{abstract}
Much research in the pathophysiology of gall stones has been devoted to various molecular species of bile salts. Recent findings have shown the importance of phospholipids in biliary pathophysiology. In the present study the addition of increasing doses of egg lecithin to human and model biles progressively prolonged the nucleation time. Concurrently biliary cholesterol was shifted from the vesicular to the non-vesicular carrier(s) while the cholesterol/phospholipid ratio of the remaining vesicles was progressively lowered. Model bile solutions of identical lipid concentration were prepared using phosphatidylcholine, phosphatidylserine, and phosphatidylethanolamine as the only phospholipid. With phosphatidylethanolamine most of the cholesterol was shifted to the vesicular carrier while phosphatidylserine shifted most of the cholesterol to the non-vesicular carrier(s). With phosphatidylcholine the cholesterol was distributed in both carriers. Phosphatidyl choline species composed of various acyl fatty acids in the sn-1 and sn-2 positions were used as the sole phosphlipid in otherwise identical model bile solutions. With palmitic acid in the sn-1 position and arachidonic acid in the sn-2 position most of the cholesterol was found in the non-vesicular carrier. When stearic acid was used in sn- 2 position instead of arachidonic acid most of the cholesterol was found in the vesicular carrier. These and other variations in phospholipid molecular species shifted cholesterol among its carriers and also modified the nucleation time of model biles. Most of these effects were also found upon addition of the various phospholipid species to human biles. These findings show the importance of phospholipid species in biliary pathophysiology and may be useful when trying to manipulate cholesterol carriers and solubility in bile.
\end{abstract}

(Gut 1993; 34: 110-115)

The aetiology of cholesterol gall stones has not been completely elucidated. Research interest during the last two decades has been focused on the metabolism of biliary lipids and its effect on gall stone formation. It has become evident that a disturbed metabolism of cholesterol and/or bile salts is a major factor in gall stone formation. ${ }^{12}$
The role of phospholipids in this process is only now beginning to be appreciated. ${ }^{3-5}$ It has been reported ${ }^{67}$ that the addition of minute amounts of phosphatidylcholines normalised the short nucleation time of bile from patients with cholesterol gall stones while having only a minimal effect on the cholesterol saturation index. Similar amounts of bile salts did not prolong the nucleation time. ${ }^{8}$ In the present study we have analysed the effect of various phospholipids and different molecular species of lecithin on cholesterol distribution between its carriers in bile and their effect on the nucleation time.

\section{Methods}

BILES

Six gall bladder bile samples (Table I, biles 2-7) were obtained at cholecystectomy and one hepatic bile (Table I, bile no 1) from a patient with an indwelling $T$ tube. All patients had cholesterol gall stones. Data on these biles are shown in Table I. All patients gave their informed consent to bile sampling.

\section{MATERIALS}

The following synthetic molecular species of phospholipids with identical fatty acid composition (sn-1 palmitoyl sn-2 oleoyl) were used: phosphatidylserine, phosphatidylethanolamine, and phosphatidylcholine. The following molecular species of phosphatidylcholine: $s n-1$ palmitoyl sn-2 stearoyl, sn-1 palmitoyl sn-2 oleoyl, sn-1 palmitoyl sn-2 arachidonoyl and sn-1 stearoyl sn-2 oleoyl were used. All were purchased from Avanti Polar Lipids, Inc (Birmingham, AL). The synthetic phospho-

TABLE I Composition of biles

\begin{tabular}{|c|c|c|c|c|c|c|c|}
\hline & CHOL & $P L$ & $B S$ & $\begin{array}{l}\text { Total } \\
\text { lipid }\end{array}$ & $C S I$ & $\begin{array}{l}\text { Nucleation } \\
\text { time }\end{array}$ & $\begin{array}{l}\% \text { Cholesterol } \\
\text { in vesicles }\end{array}$ \\
\hline \multicolumn{5}{|c|}{-- mole $\%--(\mathrm{g} / \mathrm{dl})$} & $\%$ & days & $\%$ \\
\hline 1 & $17 \cdot 2$ & $21 \cdot 7$ & $61 \cdot 1$ & 0.8 & 461 & 3 & 68 \\
\hline 2 & $14 \cdot 0$ & $22 \cdot 0$ & $64 \cdot 0$ & $5 \cdot 1$ & 210 & 3 & 24 \\
\hline 3 & 8.9 & $18 \cdot 8$ & $72 \cdot 3$ & $9 \cdot 4$ & 137 & 7 & 13 \\
\hline 4 & $18 \cdot 9$ & $23 \cdot 4$ & $57 \cdot 8$ & $2 \cdot 7$ & 298 & 6 & 47 \\
\hline 5 & $10 \cdot 4$ & $27 \cdot 2$ & $62 \cdot 4$ & $3 \cdot 4$ & 154 & 1 & 13 \\
\hline 6 & $7 \cdot 6$ & $18 \cdot 0$ & $74 \cdot 4$ & $11 \cdot 3$ & 117 & 4 & 0 \\
\hline 7 & $4 \cdot 8$ & $15 \cdot 3$ & 79.9 & $11 \cdot 8$ & 83 & 6 & 0 \\
\hline
\end{tabular}

CHOL - cholesterol, PL - phospholipid, BS - bile salts, CSI cholesterol saturation index. 
lipids and phosphatidylcholine gave a single spot by thin layer chromatography and were more than $98 \%$ pure by gas liquid chromatography of their fatty acid methyl esthers. All other lipids were purchased from Sigma Chemical Co. The egg lecithin used contained five different fatty acids.

\section{METHODS}

(1) Model biles were prepared by a modification of the method described by Kibe et al..$^{910}$ Measured aliquots of lipid stock solutions were used to produce a model bile with the desired composition: cholesterol $-10 \mathrm{~mol} \%$, bile salt (sodium taurocholate) - $72 \mathrm{~mol} \%$, phospholipid (egg yolk lecithin) - $18 \mathrm{~mol} \%$, total lipid concentration $-8.0 \mathrm{~g} / \mathrm{dl}$.

(2) The handling of native and model biles, cholesterol labelling and chromatographic separation to vesicular and non-vesicular phases were performed as described previously..$^{5111}$ The dimensions of the chromatographic column were $10 \times 330 \mathrm{~mm}$ and the flow rate was $0.7 \mathrm{ml} /$ min. The percentage of biliary cholesterol carried by the vesicular and non-vesicular fractions was calculated by measuring the area under their respective peaks, as a proportion of the total area under the cholesterol radioactivity curve eluted from the chromatographic column. ${ }^{10}$ The use of radioactive markers was previously validated by comparison with endogenous lipids. ${ }^{11}$ The fractions of the isolated vesicular and nonvesicular peaks, obtained by chromatographic separation of biles, were pooled for chemical analysis.

(3) Nucleation time was determined as described by Holan et $a l,{ }^{12}$ using a Carl Zeiss microscope equipped with a polarising system. Samples were kept for up to 30 days at $37^{\circ} \mathrm{C}$ under nitrogen and studied daily in search of cholesterol crystals.

(4) Analytic procedures: bile acid concentration was determined enzymatically. ${ }^{13}$ For the analysis of cholesterol and phospholipids the methods of Abbel et al ${ }^{14}$ and Bartlett ${ }^{15}$ were used. phosphatidylethanolamine sn-1 palmitoyl sn-2 oleoyl PS - 1-pal 2-ol= phosphatidylserine sn-1 palmitoyl sn-2 oleoyl).

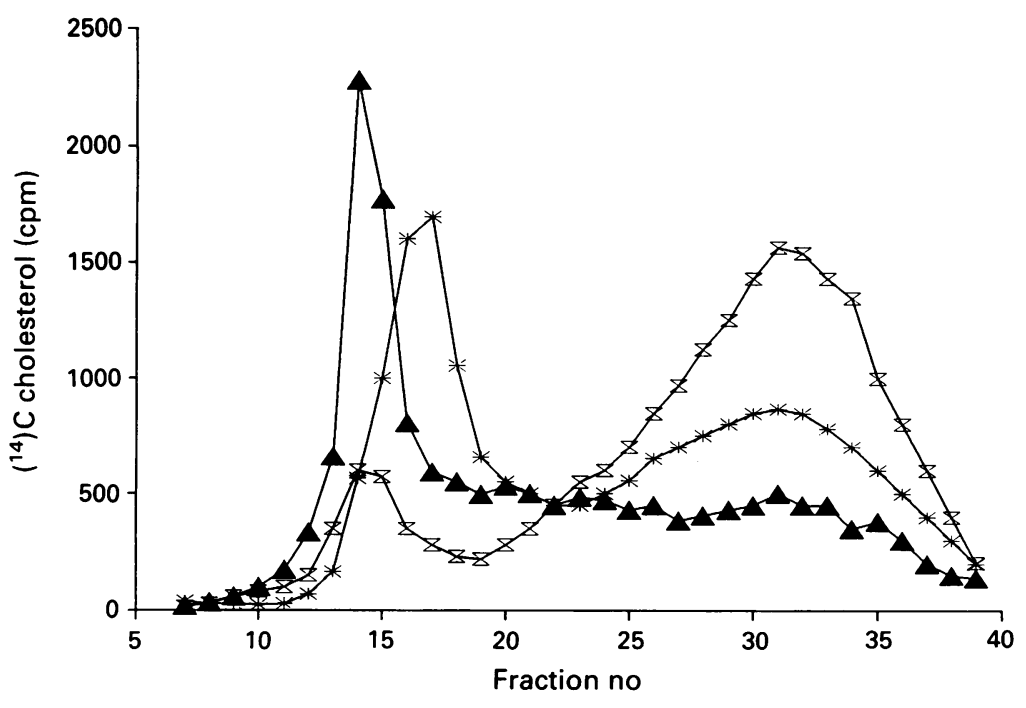

$\rightarrow$ PC 1-PAL 2-OLE $\longrightarrow$ PE 1-PAL 2-OLE

$\approx$ PS 1-PAL 2-OLE
EXPERIMENTAL DESIGN

(1) Addition of egg lecithin to native and model biles. Aliquots of egg lecithin were placed into a glass test tube and flushed to dryness under a stream of nitrogen at $37^{\circ} \mathrm{C}$, and subsequently lyophylised for 18 hours (biles 3-7). One millilitre of native or model bile samples were added to the dried lecithin and shaken at $37^{\circ} \mathrm{C}$ for one hour. After one hour samples of the bile were taken for nucleation studies and chromatographic separation. Solubilisation of egg lecithin by this process was tested with radiolabelled phospholipid. At one hour solubilisation was $\geq 90 \%$ and did not increase after further incubation for up to 24 hours. There was also no difference in solubilisation between $1 \mu \mathrm{mol} / \mathrm{ml}$ and $20 \mu \mathrm{mol} / \mathrm{ml}$. Mixing experiments adding egg lecithin were performed with seven human biles (six gall bladder, one hepatic, Table I) and three model biles.

(2) The effect of synthetic phospholipids and different molecular species of phosphatidylcholine. Model biles were prepared with the same lipid concentration as above but with different phospholipids. Each experimental data point in Figures 1 and 2 is the average of three experiments in three identical model biles.

Addition of synthetic phosphatidylcholine to native biles was performed as described above.

\section{STATISTICAL ANALYSIS}

The Student's paired $t$ test was used to compare the nucleation time experiments, the per cent of cholesterol in the vesicular phase and the cholesterol/phospholipid ratios of native biles after the addition of phospholipids.

\section{Results}

Figure 5 shows the effect of the addition of egg lecithin on the nucleation time of seven human biles. Increasing amounts of egg lecithin progressively prolonged the nucleation time. After the addition of $1 \mu \mathrm{mol} / \mathrm{ml}$ of lecithin, the nucleation time was lengthened in only one bile and after $2.5 \mu \mathrm{mol} / \mathrm{ml}$ in only two biles. After $5 \mu \mathrm{mol} / \mathrm{ml}$ the nucleation time increased considerably in five of seven biles $(p<0.05)$. Similar results were seen in the three model biles. Figure 6 shows the percentage of biliary cholesterol in the vesicular phase as a function of the amount of lecithin added. The addition of lecithin progressively shifted cholesterol to the non vesicular phase. This phenomenon was more marked in the three model biles with few vesicles remaining after the addition of $10 \mu \mathrm{mol} / \mathrm{ml}$ of lecithin. This shift was accompanied by a progressive decrease in the cholesterol/phospholipid ratio of the remaining vesicles. Below a ratio of 0.5 vesicles become unstable in the chromatographic milieu and cannot be detected (unpublished). The cholesterol/phospholipid ratio of the nonvesicular phase remained stable upon the addition of egg lecithin. Three gall bladder biles and three model biles were analysed and the data for the human biles are shown in Figure 3.

In order to investigate the effect of different phospholipids on cholesterol solubility and the 


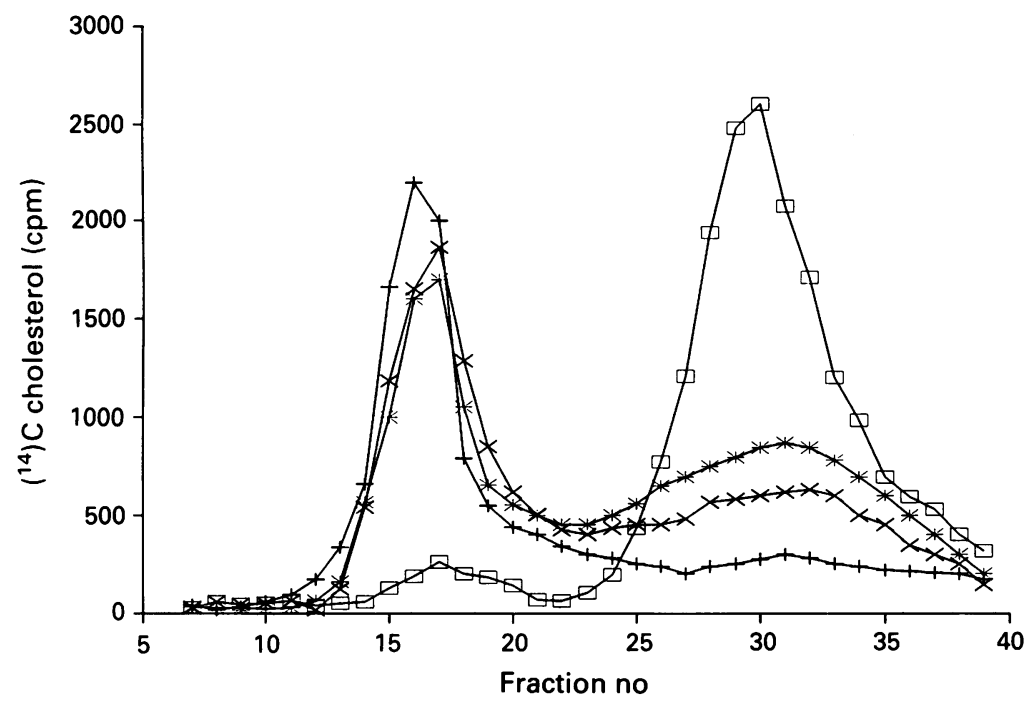

— 1-PAL 2-STE $\square$ 1-PAL 2-ARA

$\rightarrow$ 1-PAL 2-OLE $\rightarrow$ 1-STE 2-OLE

Figure 2: Elution profiles of ${ }^{14} \mathrm{C}$ cholesterol of model biles prepared with different molecular species of lecithin. (1-pal 2-ste =sn-1 palmitoyl sn-2 stearoyl, 1-pal 2-ole= sn-1 palmitoyl sn-2 oleoyl, 1-pal 2-arc=sn-1 palmitoyl sn-2 arachidonoyl, 1-ste 2-ole $=s n-1$ stearoyl $s n-2$ oleoyl).

Figure 3: Effect of egg lecithin addition on the cholesterol/phospholipid ratio of the vesicular phase in three human (Table I, biles $3-5)\left({ }^{\star} p<0.05\right)$ biles.

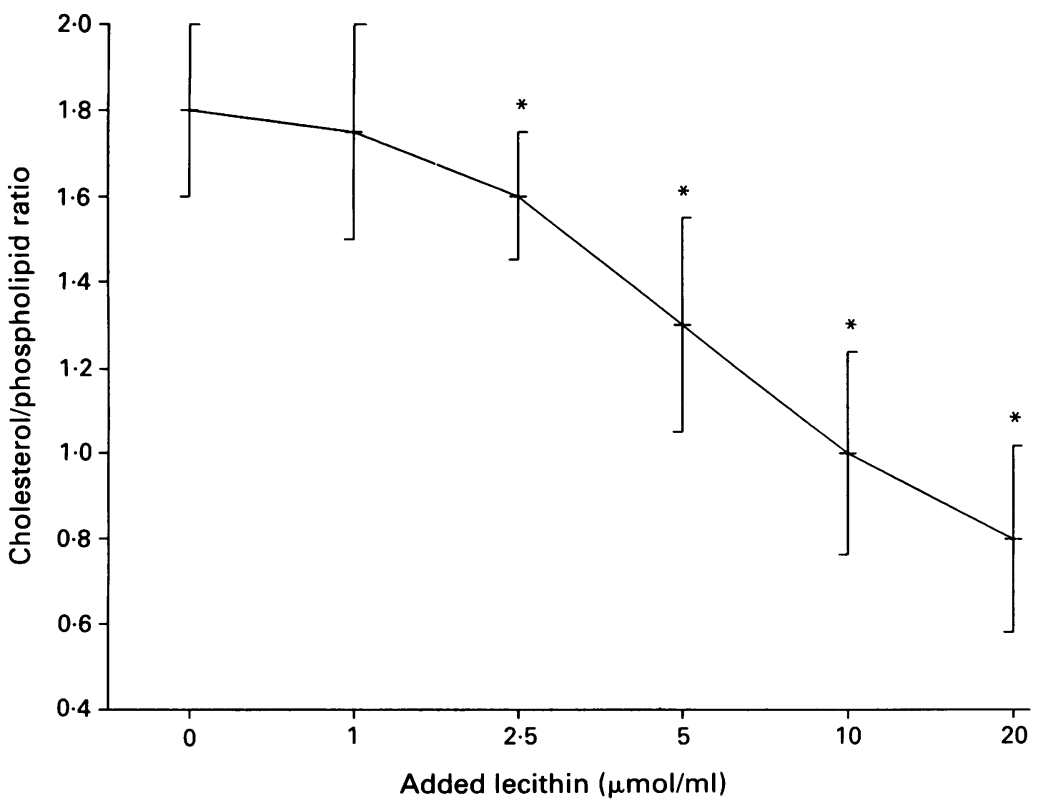

TABLE II Nucleation time of model biles prepared with different phospholipids

\begin{tabular}{lc}
\hline & $\begin{array}{c}\text { Nucleation } \\
\text { time (days) }\end{array}$ \\
\hline Phospholipid species: & \\
Phosphatidylserine sn-1 palmitoyl sn-2 oleoyl & 20 \\
Phosphatidylethanolamine sn-1 palmitoyl sn-2 oleoyl & 6 \\
Phosphatidylcholine sn-1 palmitoyl sn-2 oleoyl & 5 \\
Phosphatidylcholine molecular species: & 20 \\
Phosphatidylcholine sn-1 palmitoyl sn-2 stearoyl & 6 \\
Phosphatidylcholine sn-1 palmitoyl sn-2 oleoyl & 6 \\
Phosphatidylcholine sn-1 palmitoyl sn-2 & 5 \\
arachidonoyl & 4 \\
\hline
\end{tabular}

most of the cholesterol was in the non-vesicular phase. When (under the same conditions) the sn-2 position was occupied by a saturated fatty acid (stearic acid) almost all biliary cholesterol was found in the vesicular fraction. When the fatty acid in the sn-2 position was monounsaturated (oleic acid) cholesterol was present in both carriers though more in the vesicular fraction. Results were similar when the saturated fatty acid in sn-1 position (palmitic acid) was replaced by another saturated fatty acid (stearic acid). The nucleation time of the model biles prepared with the different phospholipids are shown in Table II. Model biles prepared with phosphatidylserine had a longer nucleation time (20 days) than those prepared with phosphatidylethanolamine or phosphatidylcholine having the same fatty acid composition. Model biles prepared with phosphatidylcholine, containing different fatty acids, had the following nucleation times: when both fatty acids (sn-1 and sn-2) were saturated (palmitic and stearic) the nucleation time was prolonged (20 days) in comparison with other phosphatidylcholines containing one unsaturated fatty acid in the sn-2 position.

Different phospholipids and different molecular forms of phosphatidylcholine were also added to three human biles. The results for one hepatic bile are shown in Figures 4 and 7 . Addition of phosphatidylethanolamine caused an almost complete shift of cholesterol to the vesicular phase. The addition of phosphatidylcholines containing various fatty acids (see methods), produced the following results: Palmitic stearic phosphatidylcholine shifted cholesterol almost completely to the vesicular phase whereas after the addition of other phosphatidylcholines biliary cholesterol remained in the non-vesicular phase. The results in the three native biles (two gall bladder and one hepatic) were similar.

\section{Discussion}

The results of this study show that the amount of phospholipids and their molecular species in bile have a decisive influence on cholesterol carriers, on the distribution of cholesterol among the carriers and on the nucleation time of bile. Jungst et al were the first to show that the addition of small amounts of phosphatidylcholine to rapidly nucleating biles (from patients with cholesterol gall stones) prolonged and normalised their nucleation time. ${ }^{6}$ The addition of similar amounts of bile salts with an equal 


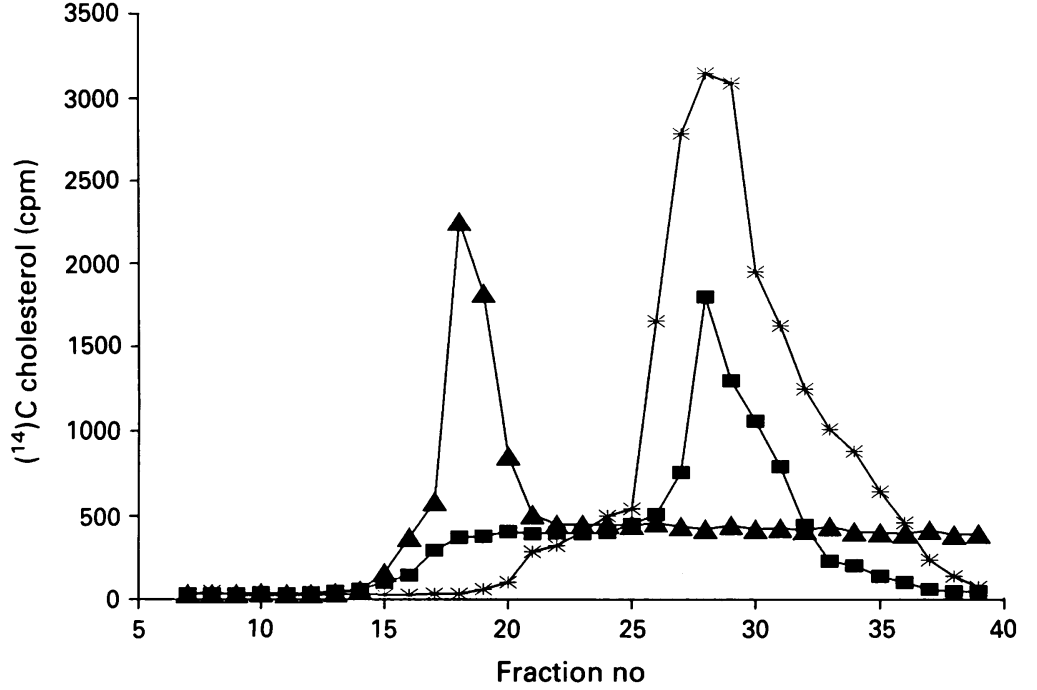

$$
\rightarrow-\text { Original bile } \rightarrow \text { PC 1-PAL 2-OLE }
$$

Figure 4: Elution profile of ${ }^{14} \mathrm{C}$ cholesterol of an hepatic bile before and after the addition of 5 umol of different phospholipids per ml of bile. Original bile composition: Chol$3 \cdot 2 \mathrm{mM}, \mathrm{BS}-18 \cdot 5 \mathrm{mM}$ $P L-6 \cdot 2 m M$.

effect on the cholesterol saturation index had no effect on the nucleation time. ${ }^{8}$

Our data show similarities and differences when compared with those of Jungst et al. ${ }^{6}$ They added 1.25 and $2.5 \mu \mathrm{mol}$ of lecithin $/ \mathrm{ml}$ bile while we added $5 \mu \mathrm{mol} / \mathrm{ml}$ bile, yet the prolongation in the nucleation time of bile was much more marked in their experiments. The nucleation time was prolonged from 1.5 days to 12.2 and 13.2 days after the addition $2.5 \mu \mathrm{mol}$ of synthetic lecithins. In our experiments the nucleation time was considerably less prolonged (Fig 5). The difference can be explained by the molecular species of lecithins used. We used egg lecithin which is a mixture of lecithins containing mostly unsaturated fatty acids in the sn-2 position. They used synthetic dipalmitoyl and distearoyl lecithins. As seen in Table II these disaturated synthetic lecithins prolonged the nucleation time to 20 days as compared with four to six days with the monosaturated lecithins.

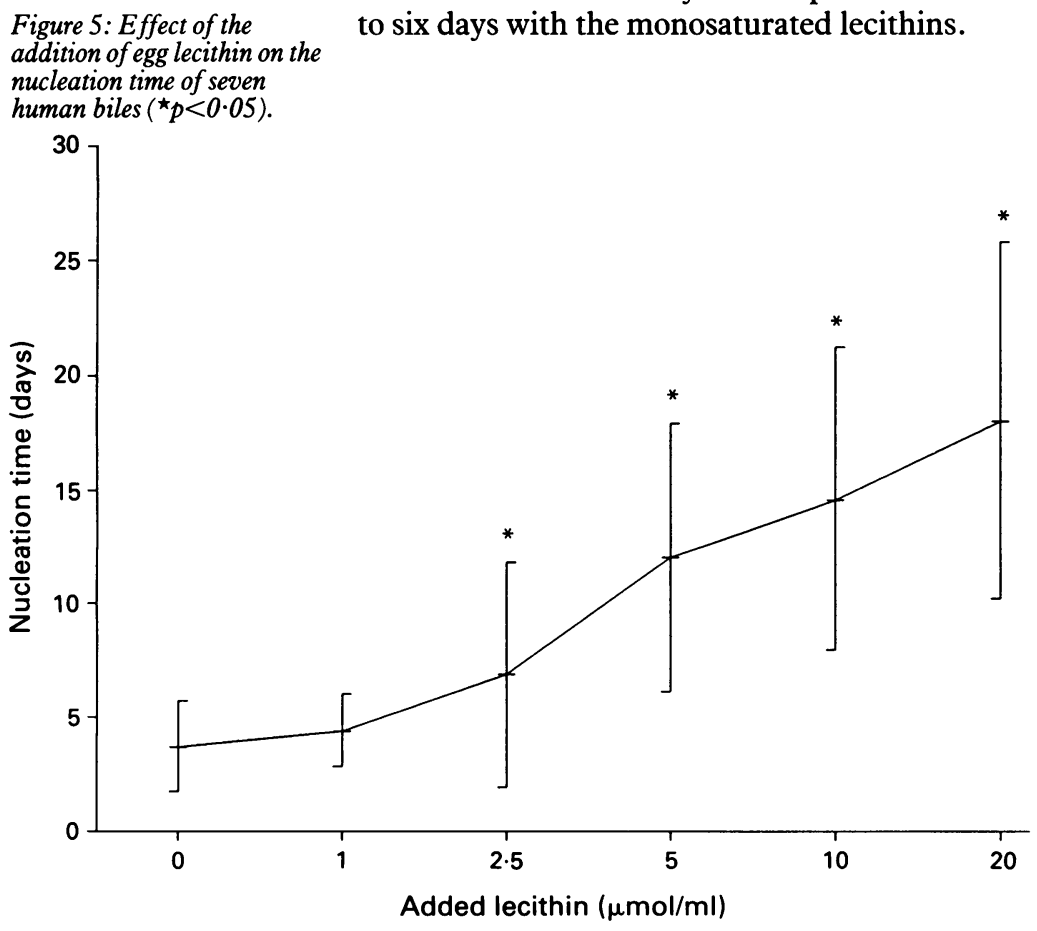

Our data show that the addition of lecithin shifts biliary cholesterol from the vesicular to the non-vesicular phase. When a similar shift is caused by the addition of bile salts or free fatty acids to bile the result is a shortening of the nucleation time. ${ }^{16}$ Phospholipids, however, prolong the nucleation time. The explanation is seen in Figure 3. The addition of egg lecithin reduced the cholesterol/phospholipid ratio of the remaining vesicles. An increase in the cholesterol/ phospholipids ratio has a very potent nucleating effect and shortens the nucleation time of model and human biles, ${ }^{17} 18$ whereas a decrease in the cholesterol/phospholipids ratio has an opposite effect. Thus the addition of phosphatidylcholine prolonged the nucleation time by two mechanisms: (A) shift of biliary cholesterol to the more stable carrier (non-vesicular) and (B) a decrease in the cholesterol/phospholipids ratio of the metastable carrier (vesicles).

Not only the total amount of phospholipids but also the molecular species has profound influences on cholesterol carriers and nucleation in bile. In order to study and characterise these differences lipid mixtures were prepared using only one species of phospholipids Some of these mixtures were definitely unphysiologic. Further studies will be needed to study the effects of various mixtures of phospholipids, as found in native bile. Using different head groups and keeping the acyl chains constant produced the following results. Phosphatidylethanolamine shifted most of the cholesterol to the vesicular phase while phosphatidylserine moved it to the non-vesicular fraction. With phosphatidylcholine, which is the major biliary phospholipid, cholesterol could be found in both carriers depending, however, on the acyl chains. With a saturated fatty acid in the sn- 1 position (palmitic acid) and the polyunsaturated arachidonic acid in the sn-2 position most of the cholesterol was in the non-vesicular phase. When under the same conditions a saturated fatty acid (stearic) replaced the arachidonic acid in the sn-2 position almost all biliary cholesterol was in the vesicular fraction. This disaturated phosphatidylcholine is, however, non-physiologic in human biles. Vesicles composed of phosphatidylethanolamine or palmitoylstearoyl phosphatidylcholine may be more rigid and this may contribute to their stability. When the fatty acid in the sn-2 position was monounsaturated oleic acid, cholesterol was present in both carriers though more in the vesicular fraction. Similar results were found when the palmitic acid in the sn-1 position was replaced by another saturated fatty acid (stearic acid). As in the vast majority of human biles the sn- 1 fatty acid is palmitic acid and physiologic variations occur mainly in the sn- 2 position, some of our data may reflect events in vivo. ${ }^{19} 20$ These findings in model biles were reproduced in human biles. The nucleation time of model biles was also markedly affected by varying the added phospholipid species. It was markedly prolonged only when both acyl groups of phosphatidylcholine were saturated (palmitic stearic). Other phosphatidylcholine species such as palmitate oleate, palmitate arachidonate and stearate oleate produced shorter nucleation time. When the acyl group composition was constant and the head 


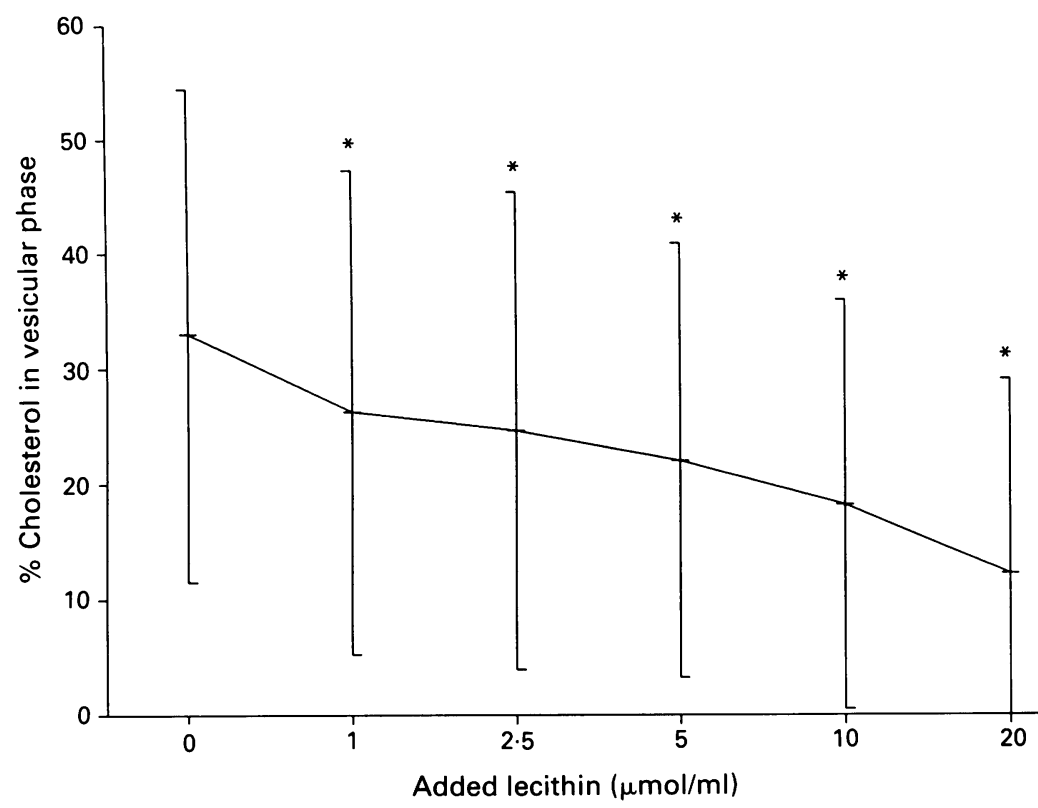

Figure 6: Effect of the addition of egg lecithin on cholesterol distribution between the vesicular and non-vesicular phases in human biles (Table I, biles $1-5)\left({ }^{\star} p<0.05\right)$.

Figure 7: Elution profile of the same hepatic bile as in Figure 4 after the addition of 5 umol of different species of phosphatidylcholine per $\mathrm{ml}$ of bile.

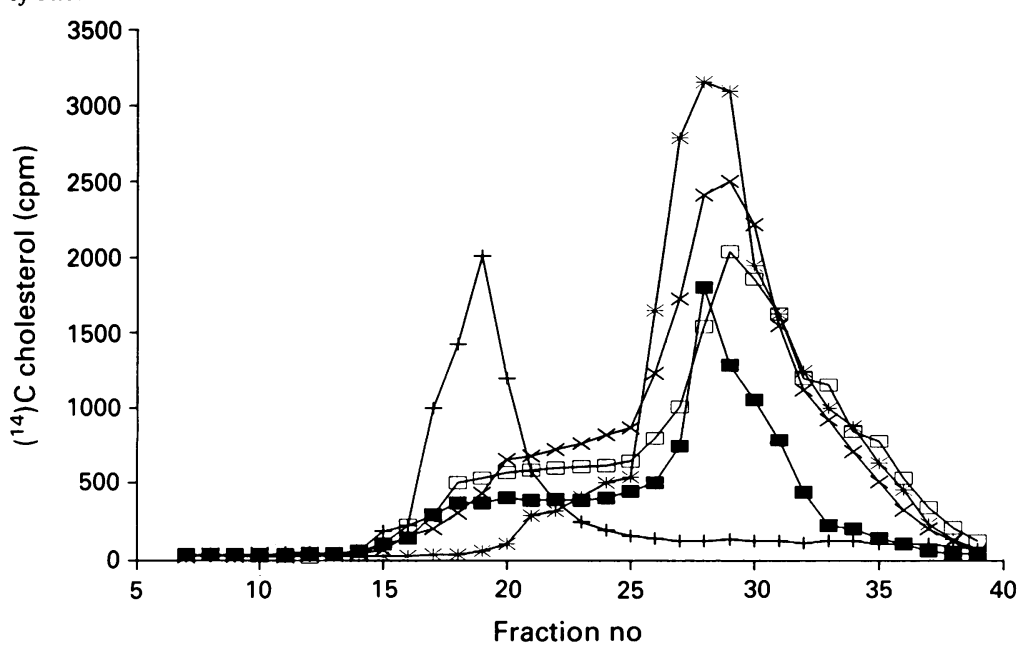

\begin{tabular}{|c|c|c|c|}
\hline$\rightarrow$ & Original bile & $\longrightarrow$ & PC 1-PAL 2-STE \\
\hline$\square$ & PC 1-PAL 2-ARA & $\rightarrow$ & PC 1-STE 2-OLE \\
\hline$\rightarrow$ & PC 1-PAL 2-OLE & & \\
\hline
\end{tabular}

bility of future therapeutic manipulation. Most of the data are new and thus cannot be compared with findings by other investigations. Recent evidence suggests that phospholipids are of major importance in biliary pathophysiology. Biliary cholesterol is probably secreted as phospholipid vesicles ${ }^{212}$ and phospholipids are probably the main cholesterol carriers in bile. ${ }^{523} \mathrm{~A}$ reduction of biliary phospholipids by dietary legumes was associated with an increased cholesterol saturation of bile..$^{24}$

The composition of phospholipids in human hepatic and gall bladder bile has been studied by a number of investigators during recent years. ${ }^{25-33}$ More than $90 \%$ of phospholipids in human bile are species of phosphatidylcholine but the presence of small amounts of other phospholipids has been observed. ${ }^{25-27}$ Seventy to $80 \%$ of biliary phosphatidylcholine is sn- 1 palmitoyl with either sn-2 oleoyl (18:1) or linoleoyl (18:2). The third most abundant phosphatidylcholine species is sn-1 palmitoyl sn-2 arachidonyl (20:4). Subjects with cholesterol gall stones were reported to have alterations in the molecular species of phosphatidylcholine in bile, although the results of various studies are conflicting. ${ }^{28-33}$ In a recent study Cohen $e t$ al working with model solutions showed a non-even distribution of different molecular species of phosphatidylcholine between the vesicular and micellar carriers. ${ }^{3+}$ Saturated fatty acids were more abundant in the vesicular phase and unsaturated ones were more abundant in the micellar phase.

In conclusion we have shown that the addition of egg lecithin to biles prolongs the nucleation time, shifts cholesterol from the vesicular phase to the non-vesicular phase and reduces the cholesterol/phospholipids ratio of the remaining vesicles. Different phospholipids and various molecular species of phosphatidylcholine markedly affect cholesterol carriers and solubility in bile. The present findings may contribute to our understanding of cholesterol solubility in bile and may be of use in our attempts to manipulate it.

1 Carey MC, Small DM. The physical chemistry of cholestero solubility in bile. $\mathcal{F}$ Clin Invest 1978; 61: 998-1026.

2 Holzbach RT, Marsh M, Olszewski M, et al. Cholestero solubility in bile. Evidence that supersaturated bile is folubility in bile. Evidence that supersaturated bile is

3 Grundy SM. Role of biliary phospholipids in cholesterol gallstone formation. Gastroenterology 1989; 96: 942-3.

4 Carey MC, Cahalane MJ. Whither bilary sludge. Gastroenterology 1989; 95: 508-23.

Somjen GJ, Marikovsky Y, Wachtel E, Harvey PRC, Rosenberg R, Strasberg SM, et al. Phospholipid lamellae are holesterol carriers in human bile. Biochim Biophys Act 1990; 1042(1): 28-35.

6 Jungst D, Lang T, Paumgartner G. Phosphatidylcholine normalize cholesterol nucleation time in gallbladder bile of patients with cholesterol gallstones. Hepatologv 1989; 10: 599.

7 Halpern Z. Biliary phospholipids. Proceeding of the international conference on gallstones and their management. Jerusalem, February 1990. (Conference proceedings.)

8 Jungst D, Huber L, Paumgartner G. Phospholipids but not bile acids normalize cholesterol nucleation time in gallbladder bile of patients with cholesterol stones. Gastrobladder bile of patients wit

9 Kibe A, Dudley MN, Halpern Z, Lynn MP, Breuer AC, Holzbach RT. Factors affecting cholesterol monohydrate crvstal nucleation time in model svstems of supersaturated bile. F L Lipid Res 1985; 26: 1102-11.

10 Peled Y, Halpern Z, Baruch R, Goldman G, Gilat T Nucleation of cholesterol from vesicular and micellar carriers in native and model biles. Hepatologv $1988 ; 8$ $91+8$.

11 Somjen GJ, Gilat T. Contribution of micellar and vesicular carriers to cholesterol solubility in bile. F Lipid Res $1985 ; 26$ 699-704. 
12 Holan KR, Holzbach RT, Hermann RE, Cooperman AM, Claffey WJ. Nucleation time: A key factor in the pathogenesis of cholesterol gallstone disease. Gastroenterology 1979; 77: 611-7.

13 Turley SD, Dietschy JM. Re-evaluation of the 3-hydroxysteroid dehydrogenase assay for total bile acids in bile. F Lipid Res 1978; 19: 924-8.

14 Abbel LL, Levy BB, Brodie BB, Kendal EE. A simplified method for the estimation of total cholesterol of total cholesterol in serum demonstration of its specificity. $7 \mathrm{Biol}$ Chem 1952; 195: 357-66.

15 Bartlett GR. Phosphorus assay by column chromatography. 7 Biol Chem 1959; 234: 466-8.

16 Halpern Z, Peled Y, Konikoff F, Goldman G, Levin I, Gilat T. Cholesterol shifts between its carriers and the nucleation time of bile. X International Bile Acid Meeting. Trends in Bile Acid Research. Freiburg: Falk Symposium No 52, 1988 .

17 Halpern Z, Dudley AM, Lynn MP, Nader JM, Breuer AC, Holzbach RT. Vesicle aggregation in model systems of supersaturated bile: Relation to crystal nucleation and lipid
composition of the vesicular phase. 7 Lipid Res 1986; 27: composition of the vesicular phase. 7 Lipid Res 1986; 27:
295-306.

18 Harvey PRC, Gilat T, Somjen G, Strasberg SM. The cholesterol/phospholipid (C/P) ratio of the vesicular cholesterol carriers in human bile and its relationship to nucleation of cholesterol monohydrate crystals. Hepatology 1986; 6: 1167

19 Angelico M, Alvaro D, Attili AF, Cantafora A. Mechanisms of secretion of biliary phosphatidylcholine: the role of bile salts. Ital f Gastroenterol 1985; 17: 278-81.

20 Alvaro D, Cantafora A, Attili AF, et al. Relationship between bile salt hydrophobicity and phospholipid composition in bile salt hydrophobicity and phospholipid composition in
bile of various animal species. Comp Biochem Physiol 1986; 83B: $551-4$.

21 Cohen DE, Angelico M, Carey MC. Quasielastic light scattering evidence for vesicular secretion of biliary lipids. Am $\mathcal{F}$ Physiol 1989; 257: G1-20.

22 Pattison NR. Solubilization of cholesterol in human bile. FEBS Lett 1985; 181: 339-42.

23 Gilat T, Halpern Z, Peled Y, Somjen G. Metastability of cholesterol carriers in bile and the pathogenesis of cholesterol gallstones. Gastroenterol Int 1990; 3: 81-6.
24 Nervi F, Cavarrubias C, Bravo P, et al. Influence of legume intake on biliary lipids and cholesterol saturation in young Chilean men Gastroenterology 1989; 96: 825-30.

25 Gottfries A, Nilsson S, Samuelsson B, Schersten T. Phospholipids in human hepatic bile, gall bladder bile and plasma in cases with acute cholecystitis. Scand F Clin Lab Invest 1968 21: $168-76$.

26 Philips GB. The lipid composition of human bile. Biochim Biophys Acta 1960; 41:361

27 Booker ML, Scott TE, LaMorte WW. Effect of dietary cholesterol on phosphatidylcholines and phosphatidylethanolamines in bile and gallbladder mucosa in the prairie dog. Gastroenterology 1989; 97: 1261-7.

28 Hay DW, Cahalane MJ, Timofeveva N, Carey MC. Molecular species of lecithins in human gallbladder bile: Hydrophilicity of the bile salt pool determines hydrophilicity of the lecithins. Hepatology 1989; 10: 599.

29 Adlberg J, Curstedt T, Einarsson K, Sjovall J. Molecular species of biliary phosphatidylcholines in gallstone species of biliary phosphatidylcholines in gallstone chenodeoxycholic acid. $\mathcal{f}$ Lipid Res 1981; 22: 404-9.

30 Cantafora A, Angelico M, DiBiase A, Pieche U, Bracci F, Attil AF, et al. Structure of biliary phosphatidylcholine in cholesterol gallstone patients. Lipids 1981; 16: 589 92.

31 Dam $\mathrm{H}$, Christensen $\mathrm{F}$. The alimentary production of gallstones in hamsters. Acta Pathol Microbiol Scand 1952; 30 $236-42$.

32 Van Berge Henegouwen GP, van der Werf SDJ, Ruben AT. Fatty acid composition of phospholipids in bile in man: promoting effect of deoxycholate on arachidonate. Clin Chim Acta 1987; 165: 27-37.

33 Tazuma S, Hatsushika S, Sagawa H, Mizuno S, Sasaki H, Tao $\mathrm{S}$, et al. Cholesterol crystal nucleation occurs in 'healthy' gallbladder bile: implication to prediction and prevention of gallbladder bile: implication to prediction and prevention of choleste

34 Cohen DE, Carey MC. Lecithin acyl chain unsaturation modulates distribution of biliary lecithin between micelles and vesicles as well as micellar cholesterol solubility and cholesterol/lecithin ratio of vesicles on bile. Gastroenterology 1990; 98: A133. 